\title{
FHIT mRNA and protein expression in hepatocellular carcinoma
}

\author{
Rajesh Kannangai ${ }^{1}$, Fikret Sahin ${ }^{1}$, Onikepe Adegbola ${ }^{1}$, Raheela Ashfaq ${ }^{2}$, Gloria H Su${ }^{1}$ \\ and Michael Torbenson ${ }^{1}$ \\ ${ }^{1}$ Department of Pathology, The Johns Hopkins Hospital, Baltimore, MD, USA and ${ }^{2}$ Department of Pathology, \\ University of Texas Southwestern, USA
}

\begin{abstract}
Loss of fragile histidine triad (FHIT) gene expression is seen in approximately $50 \%$ of hepatocellular carcinomas in China. However, little information is available on FHIT expression in hepatocellular carcinoma in the United States, where carcinogen exposure is generally lower. Investigations of FHIT mRNA in hepatocellular neoplasms and paired non-neoplastic tissues demonstrated normal-sized FHIT transcripts in all non-neoplastic tissues and in all neoplasms including 11 hepatocellular carcinomas, two fibrolamellar carcinomas, and four benign proliferative lesions. In addition, all but one malignant and all benign neoplasms showed aberrant smaller transcripts. The smaller aberrant transcripts were overexpressed in $6 / 11$ hepatocellular carcinomas and 1/2 fibrolamellar carcinomas. An additional 79 hepatocellular carcinomas, 12 fibrolamellar carcinomas and 15 hepatic adenomas were examined for FHIT expression by immunohistochemistry. No loss of immunostaining was seen in $67 / 79(85 \%)$ of hepatocellular carcinomas, while a moderate or marked decrease was seen in $12 / 79$ (15\%). Fibrolamellar carcinomas and hepatic adenomas showed no loss of FHIT expression. In conclusion, hepatocellular carcinomas retained expression of normal FHIT mRNA transcripts, but also showed universal expression of smaller sized aberrant transcripts and commonly overexpressed these aberrant transcripts. Loss of FHIT protein expression is relatively uncommon in this cohort from the United States, where exposure to hepatic carcinogens is generally low.
\end{abstract}

Modern Pathology (2004) 17, 653-659, advance online publication, 2 April 2004; doi:10.1038/modpathol.3800102

Keywords: hepatocellular carcinoma; FHIT

The recently isolated fragile histidine triad (FHIT) gene is a member of the histidine triad gene family and appears to be an important tumor suppressor gene. FHIT is located on chromosome 3p14.2, a region of high chromosomal fragility (FRA3B) that is frequently perturbed in human carcinoma. ${ }^{1}$ In particular, FHIT gene alterations have been associated with exposure to environmental carcinogenic agents. In this regard, FHIT-deficient mice have an increased frequency of spontaneous tumors and are more susceptible to exogenous carcinogenic agents. ${ }^{2}$ The way(s) in which FHIT functions as a tumor suppressor gene is (are) unknown, but FHIT protein has a proapoptotic effect when restored to FHIT protein-deficient cell lines. ${ }^{3}$ The FHIT protein is a dinucleoside $5^{\prime}, 5^{\prime \prime \prime}$ - $\mathrm{P}^{1}, \mathrm{P}^{3}$-triphosphate $\left(\mathrm{Ap}_{3} \mathrm{~A}\right)$ hydrolase $^{4}$ that produces ADP and AMP, though the tumor suppressor effect appears to be more strongly

Correspondence: M Torbenson, MD, Room B314, 1503 E Jefferson (Bond Street Building), The Johns Hopkins University School of Medicine, Baltimore, MD 21231, USA.

E-mail: mtorben@jhmi.edu

Received 4 November 2003; revised 16 January 2004; accepted 18 January 2004; published online 2 April 2004 linked to substrate binding than substrate hydrolysis. ${ }^{5}$ FHIT mRNA and protein expression is found in most human tissues and genetic alterations are found in many human carcinomas, including loss of heterozygosity and translocations. Point mutations appear to be less common. FHIT mRNA splice variants are common in carcinoma, but are also frequently found in non-neoplastic tissues from healthy individuals. ${ }^{6}$

Hepatocellular carcinoma is one of the most common carcinomas worldwide and is associated with various environmental carcinogens such as aflatoxin exposure. Previous studies have shown FHIT abnormalities in hepatocellular carcinomas at both the mRNA and protein expression levels, with aberrant mRNA transcripts and loss of protein expression in cell lines and in human hepatocellular carcinomas from China, Taiwan, and Germany. ${ }^{7,8}$. However, FHIT's role in hepatocellular carcinoma remains incompletely defined in areas traditionally associated with lower exposures to environmental carcinogens such as North America. Thus, we examined FHIT expression in a United States cohort of hepatocellular carcinomas in order to (1) determine the presence/absence of normal FHIT 
transcripts, (2) document the presence of splice variants in tumor and nontumor tissues, (3) investigate the relative expression levels of splice variants in tumor vs nontumor tissues, and (4) and examine protein expression by immunohistochemistry.

\section{Materials and methods}

\section{RNA Extraction, cDNA Synthesis}

This study was performed with appropriate Institutional Review Board approval. Fresh tissue was obtained at the time of surgery from primary liver neoplasms and adjacent non-neoplastic liver tissues. Tissue was harvested by an experienced liver pathologist, snap frozen in liquid nitrogen, and stored at $-80^{\circ} \mathrm{C}$ prior to use. For the RNA studies, 17 matched pairs of tumor and nontumor tissues were available: 11 hepatocellular carcinomas, two fibrolamellar carcinoma, one hepatic adenoma, one focal nodular hyperplasia, one benign regenerative nodule in the setting of cirrhosis from chronic hepatitis $\mathrm{C}$ infection, and one primary hepatic angiomyolipoma. The pathological diagnoses were confirmed in all cases by a liver pathologist. All tissues were obtained between 1999 and 2002 from routine surgical cases at the Johns Hopkins Hospital.

Two cell lines were also examined, HepG2 and Hep3B. Cell lines were purchased (American Type Culture Collection, Manassas, VA, USA) and propagated in Eagle minimum essential media (Fisher Scientific, Pittsburgh, PA, USA) as per American Type Culture Collection instructions. To ensure that FHIT gene defects were not introduced during repeated subcultures and freeze/thaw cycles, cell aliquots were chosen for propagation that had underwent a single subculture and freeze/thaw cycle.

RNA was extracted using TRIzol (Invitrogen Life Technologies, Carlsbad, CA, USA) followed by precipitation with isopropyl alcohol. cDNA was prepared using $1 \mu \mathrm{g}$ of RNA, oligo dT primers, and the Superscript First-Strand synthesis system for RT-PCR (Invitrogen Life Technologies, Carlsbad, CA, USA) according to the manufacturer's instructions.

\section{Gene Expression Analysis by RT-PCR and Real-Time PCR}

A measure of $1 \mu \mathrm{g}$ of cDNA was used as input for RTPCR. Primers were used as per Schlott et $a l^{9}$ to amplify exons 1-9 of the FHIT cDNA, generating a $599 \mathrm{bp}$ product (forward primer 1, 5'-CATCCTGGAAGCTTTGAAGCT-3', position-201-223; reverse primer $5^{\prime}$-TCCTCTGATCTCCAAGAGGC-3', position 379-398). PCR cycling conditions were $15 \mathrm{~min}$ at $95^{\circ} \mathrm{C}$ followed by 40 cycles of $95^{\circ} \mathrm{C}$ for $1 \mathrm{~min}, 55^{\circ} \mathrm{C}$ $1 \mathrm{~min}$, and $72^{\circ} \mathrm{C}$ for $1 \mathrm{~min}$. The amplified products were detected with ethidium bromide in $1 \%$ agarose gel. Experiments without RT polymerase showed no amplicons.
The relative expression levels of the smaller aberrant transcripts was further studied by real-time PCR, which amplified only the smaller-sized transcripts and did not detect the larger wild-type transcript. Real-time PCR was performed with the SmartCycler system (Cephid, Sunnyvale, CA, USA) using the QuantiTect SYBR green PCR kit (Qiagen, Valencia, CA, USA) as per the manufacturer's instructions with $1 \mu \mathrm{g}$ of cDNA and the same primers as above. Cycling conditions were $95^{\circ} \mathrm{C}$ for $15 \mathrm{~min}$ followed by 45 cycles of $95^{\circ} \mathrm{C}$ for $15 \mathrm{~s}, 55^{\circ} \mathrm{C}$ $30 \mathrm{~s}$, and $72^{\circ} \mathrm{C}$ for $30 \mathrm{~s}$. The specificity of PCR products were ascertained by melt-curve analysis and direct sequencing. To confirm that real-time PCR was amplifying only the smaller splice variants, the PCR products were examined by gel electrophoresis and each sample directly compared to the gel electrophoresis pattern seen on RT-PCR. To further validate the real-time PCR results, all samples were repeated with a separate assay using a new forward primer (exon 3, 5'-TCCGTAGTGCTATCTACATCC- $3^{\prime}$, position $-142-162$ ) and the same reverse primer and cycling conditions as above. Expression levels were normalized to $\beta$ glucuronidase using previously described primers. ${ }^{10}$ Dilution studies demonstrated real-time PCR efficiencies of greater than $90 \%$ for both FHIT and $\beta$-glucuronidase.

\section{FHIT Immunohistochemistry}

Of the 11 hepatocellular carcinomas that were used in mRNA expression studies, seven had matched formalin-fixed paraffin-embedded tissues from both the tumor and adjacent nontumor tissues, as did both fibrolamellar carcinomas. In order to study a greater number of hepatocellular carcinomas for protein expression, tissue arrays were next constructed from formalin-fixed, paraffin-embedded tissues using 79 typical hepatocellular carcinomas different than those studied for mRNA expression. The arrays were designed to include paired nonneoplastic and neoplastic liver tissue from each patient. Each case had at least four $1.5 \mathrm{~mm}$ cores of tumor and four $1.5 \mathrm{~mm}$ cores of non-neoplastic liver. Control tissues from diverse organs were also included (generally 10-18 cores of different types of tissue per block). The arrays were also constructed with four cases present twice on two different blocks to serve as an internal control for reproducibility of staining. In all, 15 hepatic adenomas with paired non-neoplastic tissues along with 12 fibrolamellar carcinomas were also included on the arrays.

A polyclonal rabbit anti-FHIT antibody designed to detect the full-length FHIT protein $(17 \mathrm{kDa})$ was used for immunostaining (Zymed Laboratories Inc., San Francisco, CA, USA). Sections $(5 \mu \mathrm{m})$ were incubated with the primary antibody at a 1:200 dilution for $1 \mathrm{~h}$ at room temperature. Following the primary antibody, the sections were incubated for 
30 min in Dako EnVision + Peroxidase, a labeleddextran polymer, followed by incubation with diaminobenzidine (DAB) for 5 min. Separate positive (normal breast acinar tissue) and negative controls (renal glomeruli, breast cancer) were appropriately negative for FHIT protein. In addition, no staining was seen when primary antibody was replaced by normal rabbit serum IgG.

Immunostaining was evaluated on a scale of $0-3$ for intensity and $0-4$ for distribution as per Hao et $a l,{ }^{11}$ with a final score obtained by multiplying the two individual scores. Scores of 9-12 were considered retained positivity, 6-8 as moderately reduced labeling, and 5 or less as marked reduction or loss of immunopositivity.

\section{Results}

\section{Demographics}

For the mRNA expression studies, the hepatocellular carcinomas were from eight males and three females with an average age of $60 \pm 12$ years. The underlying liver diseases were chronic hepatitis $\mathrm{C}$ $(N=5)$, chronic hepatitis B (two), cryptogenic cirrhosis (one), and no known liver disease (three).

For the tissue arrays, hepatocellular carcinomas were studied from 59 males and 20 females, with an average age of $57 \pm 14$ years at the time of surgical resection; median 58, range 10-85 years. The underlying liver diseases were available in 55 cases and included hepatitis C $(N=17)$, hepatitis B (nine), hepatitis $\mathrm{C}$ and $\mathrm{B}$ coinfection (three), ethanol cirrhosis (six), nonalcoholic fatty liver disease (one), primary sclerosing cholangitis (one), autoimmune cirrhosis (one), cryptogenic cirrhosis or no known liver disease (nine). The average tumor size was $6.4 \pm 4.2 \mathrm{~cm}$; median $5 \mathrm{~cm}$. The 12 fibrolamellar carcinomas were from nine females and three males with a median age of 27 years. Eight cases were primary tumors with an average size of $10 \pm 3 \mathrm{~cm}$; median $9 \mathrm{~cm}$, while the remaining cases were from metastatic disease. The hepatic adenomas were from 15 women and have been previously characterized in greater detail. ${ }^{12}$

\section{mRNA Expression}

RT-PCR demonstrated normal-sized transcripts in all normal tissues and all liver neoplasms, Table 1.
Splice variants/aberrant bands (Figure 1) were found in 12/17 non-neoplastic liver tissues corresponding to 11 hepatocellular carcinomas and one fibrolamellar carcinoma, but were not found in the non-neoplastic livers obtained from individuals with benign proliferative lesions. In contrast, aberrant transcripts were identified in nearly all neoplasms, both benign and malignant. Both HepG2 and Hep3B cell lines demonstrated a normal-sized band, indicating the presence of wild-type FHIT transcript, as well as aberrant smaller bands. The aberrant transcripts ranged in size from 190 to $550 \mathrm{bp}$ as estimated on agarose gels and one to two aberrant bands were typically seen per case. The sizes of the aberrant bands were often not identical between non-neoplastic and paired neoplastic tissues. A representative band from each size seen $(\mathrm{N}=7)$ was chosen for direct sequencing, which demonstrated deletions of exons from 4 to 8 in various combinations as described previously. ${ }^{7,9,13}$

Real-time PCR efficiently amplified smaller transcripts and was negative in those cases with no splice variants (Figure 1). In paired tumor and nontumor tissues, overexpression of FHIT splice variants was found in 6/11 hepatocellular carcinomas and 1/2 fibrolamellar carcinomas, Table 2 . Identical results were found for the 17 cases on repeat real-time PCR using a different forward primer, with the exception of two hepatocellular carcinomas (nos. 6, 7) that showed decreased expression on the first set but increased expression with the second set of primers. More of the cases with advanced fibrosis showed overexpression of aberrant bands (4/7) compared to those with no or mild fibrosis (1/4) in the background liver, Table 2.

\section{Protein Expression by Immunohistochemistry}

Of the cases used to examine mRNA expression, seven of the hepatocellular carcinomas and two of the fibrolamellar carcinomas had matched formalinfixed tissue for immunohistochemistry. The normal liver showed moderately strong diffuse cytoplasmic staining (Figure 2). No change in expression was seen in $5 / 7$ hepatocellular carcinomas, while two cases had marked decrease of FHIT protein. No correlation was seen between immunostaining and presence of wild type or aberrant transcripts, an observation underscored by the two cases with

Table 1 RT-PCR expression analysis of FHIT mRNA

\begin{tabular}{lccrc}
\hline Tumor (N) & $\begin{array}{c}\text { Full-length transcript } \\
\text { in nontumor (N) }\end{array}$ & $\begin{array}{c}\text { Full-length transcript } \\
\text { in tumor (N) }\end{array}$ & $\begin{array}{c}\text { Aberrant transcripts } \\
\text { in nontumor (N) }\end{array}$ & $\begin{array}{c}\text { Aberrant transcripts } \\
\text { in tumor (N) }\end{array}$ \\
\hline Hepatocellular carcinoma (11) & 11 & 11 & 10 & 1 \\
Fibrolamellar carcinoma (2) & 2 & 2 & 0 & 1 \\
Benign proliferative lesions (4) & 4 & 4 & NA & 4 \\
HepG2 cell line & NA & 1 & NA & 1 \\
Hep3B cell line & NA & 1 & 1 \\
\hline
\end{tabular}


markedly decreased expression that had both wildtype transcripts as well as overexpression of the aberrant transcripts.

In the tissue arrays, $67 / 79(85 \%)$ of the hepatocellular carcinomas showed no loss of protein expression from that of the paired non-neoplastic livers. Interestingly, however, 11 of these cases showed more intense immunostaining than that of the paired non-neoplastic tissues (Figure 2). A moderate reduction (score 6-8) in FHIT immunolabeling

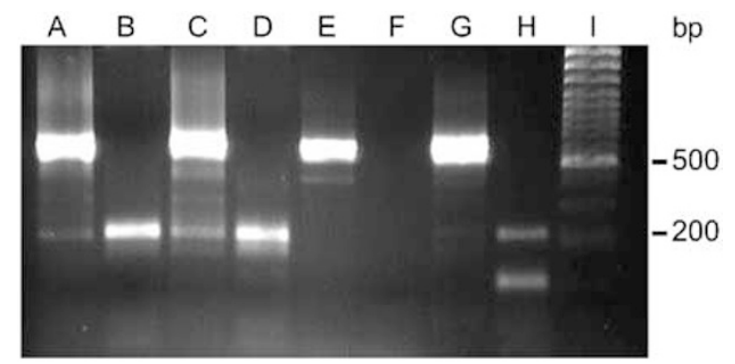

Figure 1 Typical RT and real-time PCR results for FHIT mRNA expression. The full-length FHIT transcript is $599 \mathrm{bp}$. Lane (A): HepG2 with RT-PCR; lane (B), HepG2 by real time PCR; lane (C), Hep3B by RT-PCR; lane (D), Hep3B by real-time PCR; lane (E), non-neoplastic liver by RT-PCR; lane $(\mathrm{F})$, non-neoplastic liver by real-time PCR; lane $(G)$, paired hepatocellular carcinoma by RTPCR; lane (H), paired hepatocellular carcinoma by real-time PCR; lane (I), molecular weight ladder. was seen in $8 / 79(10 \%)$ of cases while a marked reduction/loss of FHIT protein expression (score 5 or less) was seen in 4/79 (5\%) tumors, Table 3 . In contrast, no loss of FHIT staining was seen in any of the fibrolamellar carcinomas or hepatic adenomas. Among the hepatocellular carcinomas, tumors with reduced FHIT immunostaining were somewhat larger on an average than those with retained staining, $8 \pm 4$ vs $6 \pm 4 \mathrm{~cm}$, but was not statistically significant, $P=0.28$. There was also no clear association between reduction in FHIT staining and age, gender, underlying liver disease, or tumor grade, all $P>0.05$.

\section{Discussion}

As recently reviewed, the role of FHIT as a tumor suppressor gene has been supported by animal studies and examination of human tissue, which have shown loss of normal transcripts and decreased or loss of protein expression in carcinoma. ${ }^{14}$ The results from this study extend our understanding of FHIT by showing that in this cohort of hepatocellular carcinomas from the United States there is (1) retention of full-length mRNA transcript in most cases and only rare loss of protein expression, (2) consistent presence and frequent overexpression of splice variants, (3) no loss of protein

Table 2 FHIT real-time PCR and immunostaining results for 17 primary hepatic neoplasms

\begin{tabular}{|c|c|c|c|c|c|}
\hline Tumor & Age/gender & Underlying liver disease & $\begin{array}{l}\text { Tumor size } \\
(\mathrm{cm}) / \text { grade }\end{array}$ & $\begin{array}{c}\text { Fold change in } \\
\text { aberrant transcripts }\end{array}$ & $\begin{array}{c}\text { FHIT immunostain } \\
\text { score }\end{array}$ \\
\hline \multicolumn{6}{|l|}{ Hepatocellular carcinoma } \\
\hline 1 & $77 / \mathrm{M}$ & Hepatitis B cirrhosis & 3/well & 11.5 & 4 \\
\hline 2 & $59 / F$ & Hepatitis B cirrhosis & $2.5 / \bmod$ & 2.5 & 12 \\
\hline 3 & $54 / \mathrm{F}$ & Cryptogenic cirrhosis & 4/well & 1 & 12 \\
\hline 4 & $65 / \mathrm{M}$ & Hepatitis C cirrhosis & 1/well & 1.5 & 12 \\
\hline 5 & $67 / \mathrm{M}$ & Hepatitis C cirrhosis & $4 / \bmod$ & 2.5 & 2 \\
\hline 6 & $61 / \mathrm{M}$ & Hepatitis C cirrhosis & 4.4/well & 0.3 & 9 \\
\hline 7 & $40 / \mathrm{M}$ & Hepatitis C cirrhosis & $5.5 / \bmod$ & 0.1 & $\mathrm{NA}^{\mathrm{b}}$ \\
\hline 8 & $66 / \mathrm{M}$ & $\begin{array}{l}\text { Hepatitis } \mathrm{C} \text { with mild } \\
\text { fibrosis }\end{array}$ & $4.7 / \mathrm{mod}$ & 0.11 & NA \\
\hline 9 & $73 / \mathrm{M}$ & None & $2.5 / \mathrm{mod}$ & 4.1 & NA \\
\hline 10 & $70 / \mathrm{M}$ & None & $6.5 / \mathrm{mod}$ & 0.03 & 12 \\
\hline 11 & $40 / \mathrm{F}$ & None & $3.7 / \mathrm{mod}$ & 11.9 & NA \\
\hline \multicolumn{6}{|c|}{ Hepatoblastoma cell lines } \\
\hline HepG2 & NA & NA & - & Ct $36^{\mathrm{a}}$ & NA \\
\hline Нер3В & NA & NA & - & Ct 34 & NA \\
\hline \multicolumn{6}{|l|}{ Fibrolamellar carcinoma } \\
\hline 1 & $26 / \mathrm{F}$ & None & 8/well & Tumor Ct 45 & 12 \\
\hline 2 & $42 / \mathrm{M}$ & None & 15/well & 3.2 & 9 \\
\hline \multicolumn{6}{|l|}{ Benign hepatic tumors } \\
\hline Hepatic adenoma & $43 / \mathrm{F}$ & None & 4.5 & Tumor Ct 39 & NA \\
\hline Focal nodular & $45 / \mathrm{F}$ & None & 4 & Tumor Ct 39 & NA \\
\hline \multicolumn{6}{|l|}{ hyperplasia } \\
\hline Angiomyolipoma & $52 / \mathrm{F}$ & None & 4 & Tumor Ct 37 & NA \\
\hline Regenerative nodule & $52 / \mathrm{F}$ & Hepatitis C cirrhosis & 2.5 & Tumor Ct 40 & NA \\
\hline
\end{tabular}

\footnotetext{
${ }^{\mathrm{a}}$ For cases in which the non-neoplastic tissue was negative by real-time PCR and for cell lines, the threshold cycle (Ct) of the tumor is given.

${ }^{\mathrm{b}} \mathrm{NA}$, tissue not available for immunohistochemistry.
} 

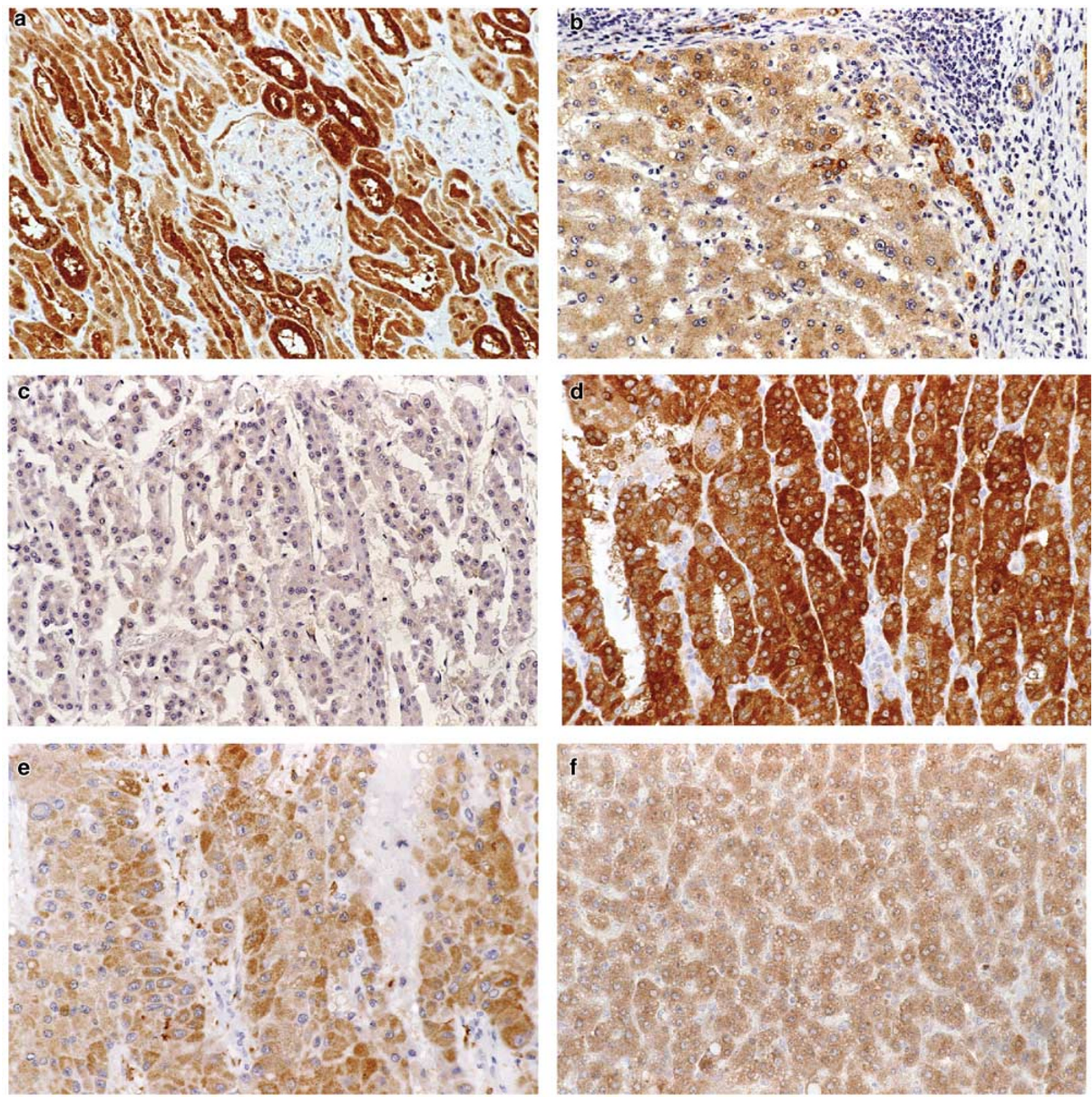

Figure 2 Immunohistochemistry for FHIT. Renal tubules are strongly positive, while glomeruli epithelial cells are negative. Weaker staining can be seen in Bowman's capsule and the mesangial cells within the glomeruli (panel (a)). Normal liver shows moderate and diffuse cytoplasmic positivity (b). A hepatocellular carcinoma shows complete loss of FHIT immunostaining (c). Another hepatocellular carcinoma shows increased cytoplasmic staining for FHIT (d). A fibrolamellar carcinoma shows retained cytoplasmic positivity (e). A hepatic adenoma also shows no loss of cytoplasmic FHIT positivity (f).

Table 3 Immunostaining patterns of FHIT protein by immunohistochemistry

\begin{tabular}{|c|c|c|c|}
\hline Tumor $(\mathrm{N})$ & $\begin{array}{l}\text { No loss of FHIT staining, } \\
\text { score }>8 \mathrm{~N}(\%)\end{array}$ & $\begin{array}{c}\text { Decreased FHIT staining, } \\
\text { score } 6-8 \mathrm{~N}(\%)\end{array}$ & $\begin{array}{c}\text { Marked decrease/loss } \\
\text { of FHIT staining, score } \\
<6 \mathrm{~N}(\%)\end{array}$ \\
\hline Hepatocellular carcinoma (79) & $67(85 \%)$ & $8(10 \%)$ & $4(5 \%)$ \\
\hline Fibrolamellar carcinoma (12) & $12(100 \%)$ & 0 & 0 \\
\hline Hepatic adenomas (15) & $15(100 \%)$ & 0 & 0 \\
\hline
\end{tabular}


expression in fibrolamellar carcinoma or hepatic adenomas.

We were able to routinely detect full-length FHIT transcripts in all studied hepatocellular carcinomas and in two hepatoblastoma cell lines, indicating preservation of at least one allele in these cases. Similarly, full-length transcripts have been found in nearly all hepatocellular carcinoma cell lines and primary tumors examined by most investigators. ${ }^{7,9,13}$ Interestingly, despite the presence of full-length transcripts, many hepatocellular carcinomas show no, or markedly, reduced protein expression. In this regard, the potential role of loss of heterozygosity remains unclear, as it has been observed variably in $0 / 18^{7}$ and 10/34 cases $^{9}$ from Asia and 2/7 cases from Italy. ${ }^{13}$

Aberrant splice variants of FHIT mRNA are widely found in tumors and can also be found in benign tissues. ${ }^{6}$ Our results confirm the common presence of the splice variants in non-neoplastic liver and demonstrate frequent overexpression in hepatocellular carcinoma. The significance of this observation is not clear as the transcripts lack exon 5 and thus presumably are not translated. Other aberrant transcripts that include exon 5 can be translated, at least in vitro, ${ }^{1}$ though in vivo translation has not been well studied. The factors related to production of aberrant transcripts are not well understood. In a study of lung carcinomas, Sato et $a 1^{15}$ reported that the prevalence of aberrant FHIT transcripts was associated with tumor stage, telomerase activity, and assay conditions, but was not associated with FHIT allelic loss. In other studies, aberrant transcripts have been found in $46-70 \%$ of hepatocellular carcinomas ${ }^{7,9,13}$ as well as in focal nodular hyperplasias ${ }^{9}$ and normal liver tissues. ${ }^{9}$ Thus, the biological relevance of the aberrant transcripts we and others have identified in hepatocellular carcinoma is not at all clear and they may in fact be irrelevant, representing only background 'noise' in the complex transcriptome of hepatocellular carcinoma.

This study is the first, to our knowledge, to report overexpression of FHIT protein in hepatocellular carcinomas by immunohistochemistry. While overexpression of a tumor suppressor gene may be somewhat counterintuitive, such a finding has been reported for other tumor suppressor genes, such as SMAD $4^{16,17}$ and may reflect interruption of a signaling/metabolic pathway downstream from the target gene. Interestingly, Ramp et $a l^{18}$ found that levels of FHIT protein in renal cell carcinomas were decreased in all cases, but were higher in some poorly differentiated carcinomas than in the welldifferentiated carcinomas. We also do not know if the overexpression of FHIT seen in our study reflects wild type or a splice variant mRNA. Kisielewski et $a 1^{19}$ have reported expression of a putative truncated FHIT protein in 3/9 cell lines by Western blot and also found overexpression of a truncated FHIT protein in a primary carcinoma.
Marked reduction or absence of FHIT protein by immunohistochemical staining has been reported in $65 \%$ of 83 hepatocellular carcinomas examined from China, ${ }^{20}$ where loss was associated with increasing tumor size and stage. In another protein expression study, $50 \%$ of 10 primary hepatocellular carcinomas from China showed loss of FHIT protein expression. In contrast, a much lower frequency of FHIT protein loss was seen in hepatocellular carcinoma in this study, which may reflect the lower exposure to environmental carcinogens in the United States.

In conclusion, hepatocellular carcinomas in this cohort retained normal FHIT mRNA transcripts in all cases, but consistently overexpressed abnormal transcripts. By immunohistochemistry, most hepatocellular carcinomas retained cytoplasmic FHIT positivity, while $15 \%$ showed moderate to marked reduction in protein expression. These findings suggest a relatively minor role for loss of FHIT expression in hepatocarcinogenesis in the United States, in contrast to the more important role for FHIT loss in hepatocellular carcinomas observed in those areas of the world with higher levels of exposure to hepatic carcinogens.

\section{References}

1 Ohta M, Inoue H, Cotticelli MG, et al. The FHIT gene, spanning the chromosome 3p14.2 fragile site and renal carcinoma-associated $\mathrm{t}(3 ; 8)$ breakpoint, is abnormal in digestive tract cancers. Cell 1996;84:587-597.

2 Zanesi N, Fidanza V, Fong LY, et al. The tumor spectrum in FHIT-deficient mice. Proc Natl Acad Sci USA 2001;98:10250-10255.

3 Roz L, Gramegna M, Ishii H, et al. Restoration of fragile histidine triad (FHIT) expression induces apoptosis and suppresses tumorigenicity in lung and cervical cancer cell lines. Proc Natl Acad Sci USA 2002; 99:3615-3620.

4 Barnes LD, Garrison PN, Siprashvili Z, et al. FHIT, a putative tumor suppressor in humans, is a dinucleoside $5^{\prime}, 5^{\prime \prime \prime}$-P1,P3-triphosphate hydrolase. Biochemistry 1996;35:11529-11535.

5 Trapasso F, Krakowiak A, Cesari R, et al. Designed FHIT alleles establish that FHIT-induced apoptosis in cancer cells is limited by substrate binding. Proc Natl Acad Sci USA 2003;100:1592-1597.

6 Panagopoulos I, Thelin S, Mertens F, et al. Variable FHIT transcripts in non-neoplastic tissues. Genes Chromosomes Cancer 1997;19:215-219.

7 Chen YJ, Chen PH, Chang JG. Aberrant FHIT transcripts in hepatocellular carcinomas. Br J Cancer 1998; 77:417-420.

8 Yuan BZ, Keck-Waggoner C, Zimonjic DB, et al. Alterations of the FHIT gene in human hepatocellular carcinoma. Cancer Res 2000;60:1049-1053.

9 Schlott T, Ahrens K, Ruschenburg I, et al. Different gene expression of MDM2, GAGE-1, -2 and FHIT in hepatocellular carcinoma and focal nodular hyperplasia. Br J Cancer 1999;80:73-78.

10 Hung CJ, Ginzinger DG, Zarnegar R, et al. Expression of vascular endothelial growth factor-C in benign and 
malignant thyroid tumors. J Clin Endocrinol Metab 2003;88:3694-3699.

11 Hao XP, Willis JE, Pretlow TG, et al. Loss of fragile histidine triad expression in colorectal carcinomas and premalignant lesions. Cancer Res 2000;60:18-21.

12 Torbenson M, Lee JH, Choti M, et al. Hepatic adenomas: analysis of sex steroid receptor status and the Wnt signaling pathway. Mod Pathol 2002;15:189-196.

13 Gramantieri L, Chieco P, Di Tomaso M, et al. Aberrant fragile histidine triad gene transcripts in primary hepatocellular carcinoma and liver cirrhosis. Clin Cancer Res 1999;5:3468-3475.

14 Pekarsky Y, Zanesi N, Palamarchuk A, et al. FHIT: from gene discovery to cancer treatment and prevention. Lancet Oncol 2002;3:748-754.

15 Sato H, Hiyama K, Ishioka S, et al. Alternative splicing, but not allelic loss, of the FHIT gene increases with development of lung cancer. Int J Oncol 1999;15:81-88.
16 Shirota Y, Kaneko S, Honda M, et al. Identification of differentially expressed genes in hepatocellular carcinoma with cDNA microarrays. Hepatology 2001;33: 832-840.

17 Torbenson M, Marinopoulos S, Dang DT, et al. Smad4 overexpression in hepatocellular carcinoma is strongly associated with transforming growth factor beta II receptor immunolabeling. Hum Pathol 2002;33:871-876.

18 Ramp U, Caliskan E, Ebert T, et al. FHIT expression in clear cell renal carcinomas: versatility of protein levels and correlation with survival. J Pathol 2002;196: 430-436.

19 Kisielewski AE, Xiao GH, Liu SC, et al. Analysis of the FHIT gene and its product in squamous cell carcinomas of the head and neck. Oncogene 1998;17:83-91.

20 Zhao P, Song X, Nin YY, et al. Loss of fragile histidine triad protein in human hepatocellular carcinoma. World J Gastroenterol 2003;9:1216-1219. 